\title{
Electrodeposition of Thin Gold Films from an Aminosilicate Stabilized Gold Sol
}

\author{
Subramanian Bharathi, James Joseph, ${ }^{\text {a }}$ and Ovadia Lev ${ }^{\mathrm{z}}$ \\ Fredy and Nadine Herrmann School of Applied Sciences, The Hebrew University of Jerusalem, Jerusalem 91904, Israel
}

Anodic deposition of thin films (up to $0.5 \mu \mathrm{m}$ thick) of gold nanodispersion from aminosilicate stabilized gold sols is demonstrated for indium-tin oxide and platinum substrates. The films are conductive, semitransparent, and comprised of 4-6 nm gold particles each coated by a thin aminosilicate shell. Anodic deposition of stabilized gold nanoparticles is a new way to produce modified electrodes comprised of silicate-noble metal composite coatings with a high degree of interconnectivity between the gold grains. (C) 1999 The Electrochemical Society. S1099-0062(98)10-072-X. All rights reserved.

Manuscript submitted October 21, 1998; revised manuscript received January 19, 1999. Available electronically March 23, 1999.

Chemically functionalized metal nanoparticles exhibit desirable optical and catalytic properties that make them ideal building blocks for two and three-dimensional molecular/nanoarchitectures. Along this line, a rapid progress is taking place in the deposition of monolayers of conductive nanocrystals on solid supports. Natan and coworkers $^{1-3}$ laid the basis for a general methodology ${ }^{4-8}$ for the preparation of conductive electron-percolating monolayers of stabilized metal nanoparticles on solid supports. The methodology is based on pretreatment of the solid surface (such as metal, glass, and alumina) with organofunctional trialkoxysilane compounds containing amine, cyanide, thiol, or other functional anchors. Stabilized gold or silver nanoparticles are then bound to these coatings. Variants of this methodology include the use of poly(ethyleneimine) or other coated surfaces that can anchor a monolayer of stabilized metal nanoparticles. ${ }^{4-7,9}$ Despite the electronic and electrochemical significance of conductive 3-D nanometallic structures, little research work has been devoted to employing the fast growing technology of stabilized nanoparticle sols for construction of 3-D structures. Notable exceptions are Murray's ${ }^{10}$ and Natan's ${ }^{11}$ recent reports on conductive nanocrystalline 3-D structures. Murray and co-workers deposited alkanethiol stabilized gold clusters on interdigitated array electrodes and examined their electrochemistry. Natan and coworkers deposited gold colloids layer by layer utilizing bifunctional molecular bridges (e.g., alkyldithiol) between the stabilized nanocrystalline layers. Natan reported that this step by step process is time consuming. ${ }^{3}$

This report demonstrates an electrochemical method of polymerizing 3-D gold aminosilane films containing interconnected gold network. This study was influenced by the research of Murray ${ }^{12}$ and others (see Ref. 13 for a recent review) that deposited aminosilicate monolayers on ITO and on noble metal electrodes by anodic formation of oxide layers (e.g., $\mathrm{PtO}$ or $\mathrm{AuO}$ ) and subsequent bonding of aminoalkylalkoxysilane moieties on these surfaces. We have recently demonstrated ${ }^{14}$ that it is possible to stabilize gold sols in aqueous solutions by aminosilicate shells in which the amine groups are oriented toward the gold surface. These two advances paved the way for multilayer electrochemical deposition of aminosilicate modified gold particles on electrodes.

In analogy with electrochemical polymerization of polypyrrole and other conductive polymers one can anticipate significant advantages for the electrodeposition of silicate-noble metal films compared to other deposition techniques such as dip and spin coating. Perhaps the most important advantages include accurate control over the amount of the electrochemical deposit and its morphology. Additionally, electropolymerization of thin films provides a convenient way to incorporate catalysts, biocatalysts, chelating agents, redox couples, mediators, and other electrode modifiers during the deposition process. In this respect, anodic deposition of silicate stabilized metal dispersion provides a convenient way to incorporate organic and inorganic modifiers during the deposition process in

a On leave from Central Electrochemical Research Institute, Karaikudi-6, India.

z E-mail: ovadia@vms.huji.ac.il contradistinction to the conventional cathodic electrodeposition of gold. In this communication we outline a way for anodic deposition of stabilized gold particles.

\section{Experimental}

Chemicals. $-N$-[3-(trimethoxysilyl) propyl ethylenediamine], (EDAS) and $\mathrm{HAuCl}_{4}$ were obtained from Sigma Chemicals. All chemicals were of analytical grade. Double distilled water was used throughout the study. Indium-tin oxide (ITO) coated glass slides of $20 \Omega \mathrm{cm}$ resistance were purchased from M/S Delta technologies (Minnesota, USA).

Preparation of the gold sol.-Gold sols were prepared using a procedure reported earlier. ${ }^{13}$ Typically, a required amount of EDAS was dissolved in $0.1 \mathrm{M} \mathrm{KH}_{2} \mathrm{PO}_{4}$ and sonicated for $10 \mathrm{~min}$. The $\mathrm{pH}$ was then adjusted using concentrated $\mathrm{HCl}$. Later, $\mathrm{HAuCl}_{4}$ was added to this solution. ${ }^{\mathrm{b}}$ The solution was sonicated for $10 \mathrm{~min}$, after which the gold sol was formed by the addition of a few drops of $0.1 \mathrm{M} \mathrm{NaBH}_{4}$. A solution containing $1 \mathrm{mM}$ gold and $100 \mathrm{mM}$ EDAS at a pH of 4.5 was used in most of the studies presented in this paper. Absorption spectra of the gold sol showed the surface plasmon absorption peak at ca. $520 \mathrm{~nm}$ characteristic of gold sol. The size of the gold particles was determined by TEM and found to be 4-6 $\mathrm{nm}$ gold particles depending on $\mathrm{pH}$ with narrow size distribution $( \pm 1 \mathrm{~nm})$.

Substrate pretreatment.-ITO coated glass pieces were pretreated prior to the electrochemical deposition by sonication in methanol and were then rinsed in water, dipped in $0.1 \mathrm{M} \mathrm{NaOH}$ solution for 2 min, and rinsed again with distilled water. Electrodes were stored under dried conditions. Platinum foil was rinsed in nitric acid and distilled water prior to deposition.

Electrochemical measurements.-The electrochemical cell consisted of a single compartment, with a Pt wire counter electrode and an $\mathrm{Ag} / \mathrm{AgCl}$ reference electrode. ITO coated glass with an area of $0.25 \mathrm{~cm}^{2}$ served as the working electrodes. A computer controlled EG\&G potentiostat (model 263A) was used to record cyclic voltammograms and for electrodeposition. After modification the electrode was rinsed thoroughly with distilled water and dried and stored in air. All measurements were carried out after thorough rinsing of the electrode. The absorption spectra of the nanocrystalline gold films were recorded using a Cary E1 UV-visible spectrophotometer. All the spectra were recorded in air. The surface morphology and the growth pattern of the nanocrystalline films were examined using an atomic force microscope (AFM, Topometerix, USA) with a silicon tip in tapping mode.

\section{Results and Discussion}

Figure 1 presents the absorption spectrum of the gold sol prepared as described above. The surface plasmon absorption peak at ca. $520 \mathrm{~nm}$ is attributed to the gold sol. The particle size of the gold sol was determined by TEM. A typical section of a micrograph is presented in the inset of Fig. 1. Spherical gold particles with 4-6 nm diam were observed.

b EDAS-phosphate salt precipitate at high concentration. If higher concentration of phosphate is required it is recommended to add the buffer after gold reduction. 


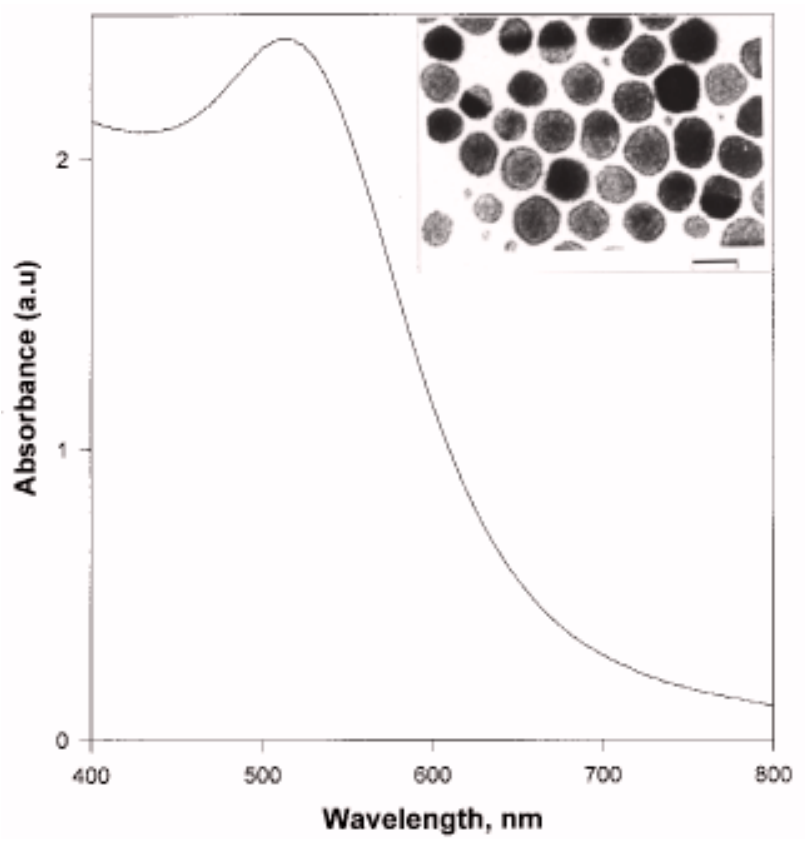

Figure 1. Absorption spectrum of gold sol prepared by the procedure described in the Experimental section and a section of a TEM micrograph of gold particles deposited on a TEM grid. (Scale bar $=6 \mathrm{~nm}$.)

The electrodeposition is carried out by cycling the electrode potential between -0.4 and $1.0 \mathrm{~V}$ vs. $\mathrm{Ag} / \mathrm{AgCl}$ at a scan rate of 100 $\mathrm{mV} / \mathrm{s}$ in the silane-stabilized sol, at $\mathrm{pH}$ 4.5. After the modification the electrode was rinsed thoroughly with distilled water and dried in a steam of nitrogen. In some cases, the electrodes were then refluxed in tetramethoxysilane for $3 \mathrm{~h}$ followed by an overnight heat-treatment at $85^{\circ} \mathrm{C}$ (or at $200^{\circ} \mathrm{C}$ for shorter duration). This treatment improved the adhesion of the gold-silicate film to the substrate without impairing its electrochemical response and optical characteristics. Figure 2 shows the absorption spectra of thin gold film deposited on ITO substrate. Wine-colored film was observed after several cycles. The plasmon excitation band could be observed even after the first few cycles and it gradually evolved during the next 200 cycles. Then, a gradual red shift of the plasmon band was observed. When the distance between the adjacent particles decreased, fluctuations in their electric field became mutually dependent thus lowering the plasmon excitation energy and red shifting the absorption band. During the multilayer deposition stage, more particles accumulated on the surface, bringing some of these particles into close proximity with one another. Hence, film growth is reflected in red shifting of the surface plasmon band. Figure 2 demonstrates that the shift of the plasmon band to $600-700 \mathrm{~nm}$ occurred gradually. This observation is somewhat different from other studies ${ }^{1}$ that demonstrated gradual evolution of a second plasmon band at ca. 600-700 $\mathrm{nm}$ in addition to the first one at $500-550 \mathrm{~nm}$. This probably indicates that the electrodeposition process gives more homogeneous distribution of the gold particles in the film. The electrochemical deposition mechanism directs the deposition of gold particles to locations that are in electric contact with the underlying ITO substrate. After more than 2000 cycles there was no further significant shift of the absorption maxima. Starting with higher concentration of gold in the solution (e.g., $10 \mathrm{mM}$ gold), the process became faster (approximately three- to fivefold) and in this case the electrode appearance became more reflective and metal-like after ca. 2000 cycles. This observation is in agreement with findings of Natan and co-workers ${ }^{11}$ that found transition to metallic behavior for thick films. We attribute this change to metallic aggregation leading to the formation of continuous gold phases.

The morphology of the thin film was also followed by AFM studies. Typical representative AFM image of the films formed after 20,

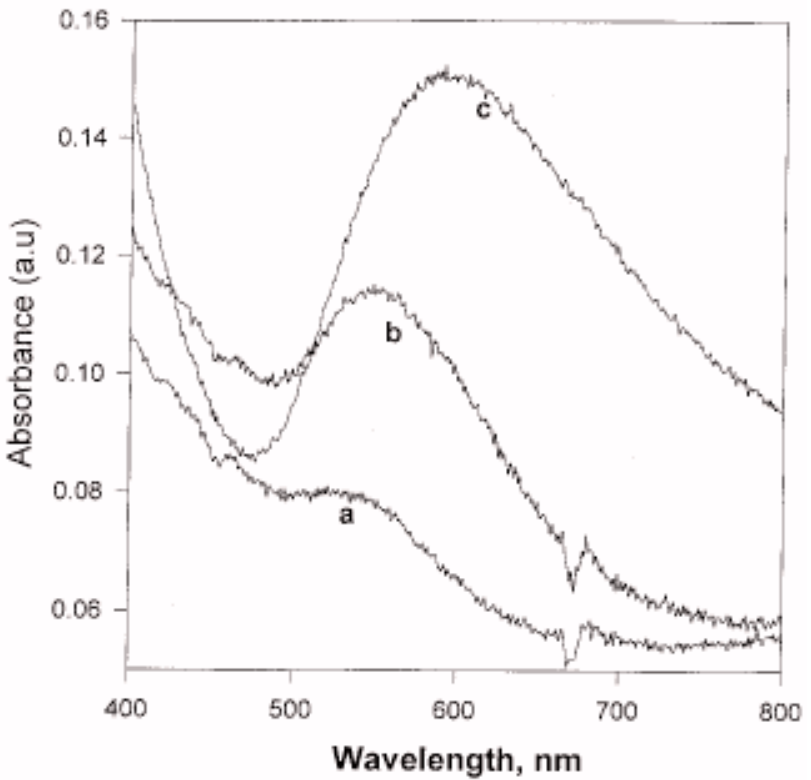

Figure 2. Absorption spectra of thin gold-aminosilane films on ITO electrode at different stages of electrodeposition (a) 20, (b) 200, and (c) 2000 cycles. The film was deposited from $0.5 \mathrm{M} \mathrm{KH}_{2} \mathrm{PO}_{4}, 100 \mathrm{mM}$ EDAS, $1 \mathrm{mM}$ Au sol, by scanning the potential in the range of -0.4 to $1.0 \mathrm{~V}$ vs. $\mathrm{Ag} / \mathrm{AgCl}$ at 100 $\mathrm{mV} / \mathrm{s}$.

200, 2000 cycles are presented in Fig. 3. The exact shape of the AFM images is influenced by the geometry of the AFM tip but the absolute heights reflect incomplete surface coverage. It can be seen that there are preferential nucleation spots creating stalagmite-like structures. The morphology of the electrodeposited film is much rougher compared to dip coated films from the same solution.

We have carried out comparative studies of electrochemically deposited films with films that were deposited by dip coating from the same sol. The X-ray photoelectron spectroscopy (XPS) studies revealed that the atomic ratio of $\mathrm{Au}: \mathrm{Si}$, calculated from characteristic $\mathrm{Au}(4 \mathrm{f})$ and $\mathrm{Si}(2 \mathrm{p})$ peaks at $83.8 \pm 0.2$ and $102.6 \pm 0.2 \mathrm{eV}$, respectively, was approximately $6: 1$. This remains the same throughout the film as shown by the depth profiling by argon spattering of the film. The Au:Si ratio of the electrodeposited film was an order of magnitude higher than that for dip coated film deposited from the same solution. Note that $\mathrm{Au}: \mathrm{Si}$ in the sol was only 1:100 which is two orders of magnitude lower as compared to the concentration of gold in the electrodeposited film. This indicates a preferential concentration of the gold by the electrodeposition technique.

We have conducted electrochemical studies in order to investigate the connectivity of the gold network. Electrochemical analysis provides the only tool that can accurately quantitate the electrochemically active surface area of the gold, which is both exposed to the solution and electronically connected to the ITO support. Underpotential copper deposition (copper UPD) ${ }^{15}$ and gold oxide formation and reduction ${ }^{16}$ were used to quantitate the active area of the gold. Figure 4 presents typical cyclic voltammetry (at $3 \mathrm{mV} / \mathrm{s}$ ) of a thin film of nanocrystalline gold particles showing the oxide formation wave and its cathodic reduction peak. Inset (A) of Fig. 4 shows the cyclic voltammetry $(3 \mathrm{mV} / \mathrm{s})$ during copper UPD from a solution containing $1 \mathrm{mM} \mathrm{CuSO}_{4}$ and $0.5 \mathrm{M} \mathrm{H}_{2} \mathrm{SO}_{4}$. The calculated charge under the gold oxide reduction peak was plotted against the number of cycles as seen in the inset (B) of Fig. 4. The charge due under the oxide reduction peak increased with increasing number of cycles reaching in this case approximately $2.8 \mathrm{mC} / \mathrm{cm}^{2}$ after 2000 cycles.

By comparing the results from XPS depth profiles (which give the total amount of gold) and the UPD of $\mathrm{Cu}$ (which gives the electrochemically active surface area), one can estimate the fraction of electrochemically active gold surface. For a film with an average 

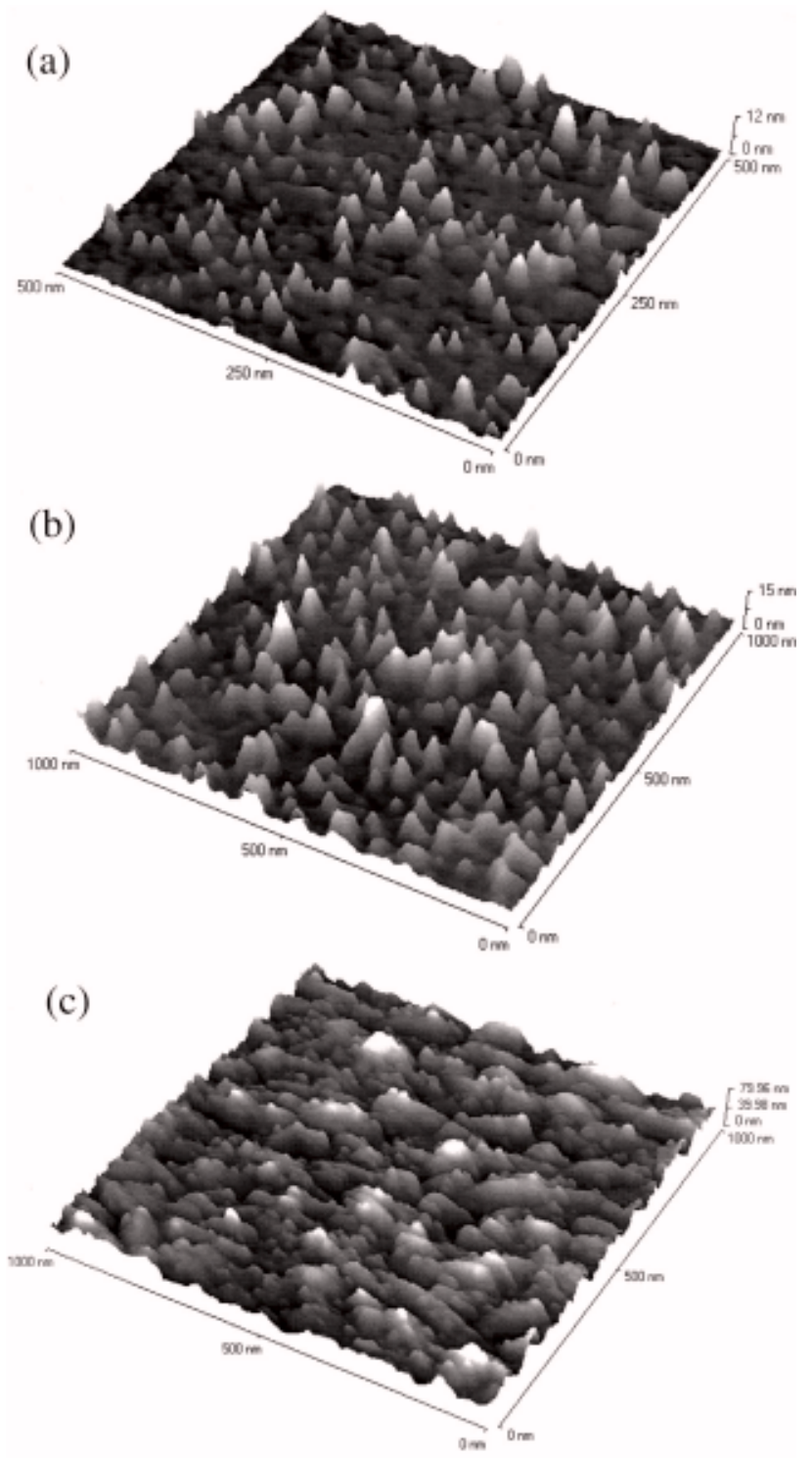

Figure 3. Tapping mode AFM image of a thin nanocrystalline film of goldaminosilane on ITO, after (a) 20 (b) 200, and (c ) 2000 cycles of electrodeposition. Solution composition as in Fig. 1.

thickness of $200 \mathrm{~nm}$ (as measured by a profilometer) it was found that approximately $20-30 \%$ of the gold surface is electrochemically active. This value points to high interconnectivity especially since some of the gold surface is covered by the aminosilane and is not accessible for electrochemical analysis. For comparison only 1-3\% of the gold is active in dip coated film of similar thickness.

In order to preclude the possibility that the process depends on the substrate and can take place only on ITO coated electrodes we have repeated the electrodeposition process on a Pt electrode. AFM imaging and gold oxide reduction tests confirmed that multilayer deposition takes place. Moreover, the deposition rate on $\mathrm{Pt}$ was much faster compared to ITO coated electrodes probably due to much denser first layer.

To confirm that the electrodeposition process is driven by the anodic polarization we have conducted several tests. First, an ITO electrode was immersed in the EDAS:Au sol overnight. In a second set of tests we repeated the electrodeposition protocol under steadystate polarization at different potentials (but with the same $\mathrm{pH}$ and the same sol composition). For the nonpolarized electrodes that were held at potentials $<0.8 \mathrm{~V} / \mathrm{Ag} / \mathrm{AgCl}$ we always observed less than a monolayer deposition even after $24 \mathrm{~h}$ of immersion in the

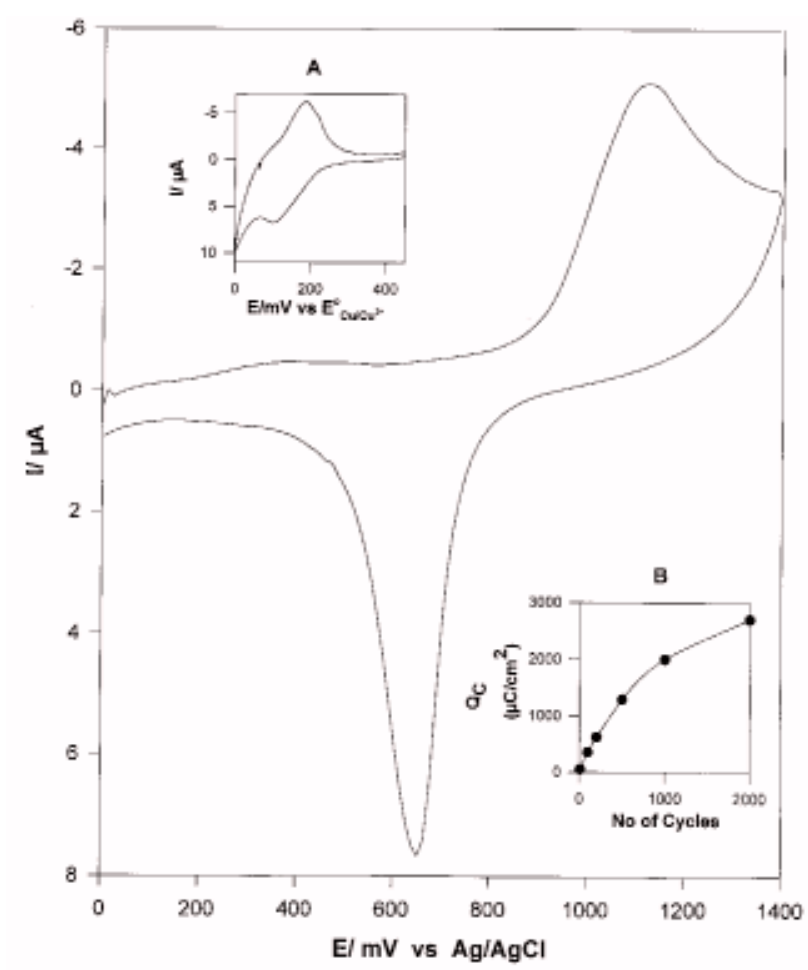

Figure 4. CV of gold-aminosilane modified ITO electrode (prepared by 2000 cycles from the same solution of Fig. 1) in $0.5 \mathrm{M} \mathrm{H}_{2} \mathrm{SO}_{4}$ (scan rate $=3 \mathrm{mV} / \mathrm{s}$ ). Inset (A): Copper UPD on the gold-silicate electrode in the presence of $1 \mathrm{mM}$ $\mathrm{CuSO}_{4}$ and $0.5 \mathrm{M} \mathrm{H}_{2} \mathrm{SO}_{4}$ (scan rate $=3 \mathrm{mV} / \mathrm{s}$ ). Inset (B): Cumulative charge for oxide reduction in films produced after the indicated number of cycles. (Electrode area $=0.25 \mathrm{~cm}^{2}$.)

EDAS: gold sol. Only for $E>1.0 \mathrm{~V}$ vs. $\mathrm{Ag} / \mathrm{AgCl}$ we observed more than one monolayer deposition. Likewise, cycling the potential of the electrode in the range of -0.4 to $0.6 \mathrm{~V}$ for $2 \mathrm{~h}$ did not result in multilayer deposition. These tests support the view that either gold oxide formation or electrostatic attraction (or both) are necessary for electrodeposition. Oxide formation can drive the condensation reaction and the positively charged surface probably helps to overcome the electrostatic barrier of the negatively charged silicate stabilized sols. ${ }^{\mathrm{c}}$

Two possible mechanisms can account for the electropolymerization. The first relates to field driven attraction of the negatively charged aminosilane coated gold particles to the positively charged electrode. Higher concentration increases the rate of polymerization. This mechanism explains the formation of the stalagmite-like structures by the lower dielectric coefficient of the gold nanoparticle stalagmites compared to aminosilane blocked sites. A second mechanism involves sequential oxidation of the deposited gold particles, which provides reactive sites for condensation reaction with the silanol shells of the gold particles. This mechanism explains also the stalagmite structure because of preference for further growth on the gold oxide. Further research is needed in order to prefer one mechanism to the other.

Another enigma relates to the preferential uptake of the gold by the film compared to its relative concentration in the sol. The amine moieties, attached to the gold surface cannot protonate and thus the aminosilicate coated gold particles are more negatively charged as compared to aminosilane moieties. Thus, the coated gold particles are attracted to the electrode more strongly than the near-neutral aminosilane moieties. We have confirmed this point by electrophoretic experiments which have demonstrated that the gold particles are more negatively charged compared to the aminosilane moi-

\footnotetext{
c The fact that the aminosilane stabilized gold sols are negatively charged at $\mathrm{pH} 4-5$
} was confirmed by gel electrophoresis. 
eties. The point of zero charge of the latter lies around $\mathrm{pH} 4.5$. This issue and the underlying mechanism for electrodeposition will be addressed separately.

\section{Conclusions}

We have demonstrated the formation of thin nanocrystalline gold films on conducting substrates (ITO and platinum). The cross-linked silicate stabilizes the film. Electrochemical processing offers considerable advantages for accurate control of the deposition parameters and thus this process may have useful practical consequences. Additionally, the process offers a new way to incorporate organic and inorganic dopants into the gold-silicate deposit during the electrochemical polymerization of nanocrystalline gold film, which may become valuable for sensing and optical applications. ${ }^{17}$

\section{Acknowledgments}

The authors gratefully acknowledge the financial support of the India-Israel collaborated research funds. The authors thank the IISc, Bangalore partners Professor Sampath and Professor Bhattacharya for fruitful collaboration. S.B. thanks the Valazzi-Pikovsky Fellowship Fund of The Hebrew University of Jerusalem for a postdoctoral fellowship.

The Hebrew University of Jerusalem assisted in meeting the publication costs of this article.

\section{References}

1. R. G. Freeman, K.C. Grabar, K. J. Allison, R. M. Bright, J. A Davis, A P Guthrie, M. B. Hommer, M. A. Jackson, P. C. Smith, D. G. Walter, and M. J. Natan, Science, 267, 1629 (1995).
2. K. C. Grabar, R. G. Freeman, M. B. Hommer, and M. J. Natan, Anal. Chem., 67, 735 (1995).

3. K. C. Grabar, K. J. Allison, B. E. Baker, R. M. Bright, K. R. Brown, R. G. Freeman, A. P. Fox, C. D. Keating, M. D. Musick, and M. J. Natan, Langmuir, 12, 2353 (1996); K. R. Brown and M. J. Natan, Langmuir, 14, 726 (1998).

4. G. Schmid, St. Peschel, and Th. Sawitowski, Z. Anorg. Allg. Chem., 623, 719 (1997).

5. J. J. Spatz, A. Roescher, and M. Moller, Adv. Mater, 8, 337 (1996).

6. A. Doron, E. Katz, and I. Willner, Langmuir, 11, 1313 (1995).

7. D. J. Schiffrin, O. A. Petrii, G. A.Tsirlina, and S. N. Pron'kin, J. Electroanal. Chem., In press.

8. K. R. Brown, A. P. Fox, and M. J. Natan, J. Am. Chem. Soc., 118, 1154 (1996).

9. G. Chumanov, K. Skolov, B. W. Gregory, and T. M. Cotton, J. Phys. Chem., 99, 9466 (1995).

10. R. H. Therill, T. A. Postlethwaite, C-h. Chen, C-D. Poon, A.Terzis, A. Chen, J. E Hutchison, M. R. Clark, G. Wignall, J. D. London, R. Superfine, M. Falvo, C. S. Johnoson, E. T. Samulski, and R. W. Murray, J. Am. Chem. Soc., 117, 12537 (1995).

11. M. D. Musick, C. D. Keating, M. H. Keefe, and M. J. Natan, Chem. Mater, 9, 1499 (1997).

12. J. R. Lenhard and R. W. Murray, J. Am.. Chem. Soc., 100, 7870 (1978).

13. O. Lev, Z. Wu, S. Bharathi, A. Modestov, V. Glezer, J. Gun, L. Rabinovich, and S. Sampath, Chem. Mater, 9, 2354 (1997).

14. S. Bharathi and O. Lev, Chem. Commun., 2303 (1997)

15. D. M. Kolb in Advances in Electrochemistry and Electrochemical Engineering, H. Gerischer and C. W. Tobias, Editors, Vol. 11, John Wiley \& Sons, New York (1978).

16. D. A. J. Rand and R. Woods, J. Electroanal. Chem., 31, 29 (1971)

17. O. Lev, M. Tsionsky, L. Rabinovich, V. Glezer, S. Sampath, I. Pankratov, and J. Gun, Anal. Chem., 67, 22A (1995). 